\title{
Cyclooxygenase-2 Activity Following Traumatic Brain Injury in the Developing Rat
}

\author{
ROBERT W. HICKEY, P. DAVID ADELSON, MICHAEL J. JOHNNIDES, DWIGHT S. DAVIS, ZHISHENG YU, MARIE E. ROSE, \\ YUE-FANG CHANG, AND STEVEN H. GRAHAM
}

\author{
Department of Pediatrics [R.W.H., M.J.J.], Department of Neurosurgery [P.D.A., D.S.D., Y.-F.C.], Department of Neurology \\ [Z.Y., M.E.R., S.H.G.], University of Pittsburgh School of Medicine, Pittsburgh, Pennsylvania 15213; Geriatric Research Education \\ and Clinical Center [S.H.G.], VA Pittsburgh Healthcare System, Pittsburgh, Pennsylvania 15206
}

\begin{abstract}
Cyclooxygenase (COX) is the rate-limiting enzyme in the production of prostaglandins. COX-2, the predominant COX isoform in brain, is induced by synaptic activity. COX-2-generated prostaglandins are important regulators for a range of activities under physiologic conditions. However, under pathologic conditions, COX-2 activity can produce reactive oxygen species and toxic prostaglandin metabolites that can exacerbate brain injury. In this study, we examine the developmental production of COX-2 and test the ability of a COX-2 inhibitor, SC58125, to attenuate traumatic brain injury in developing rats. We show that constitutive COX-2 concentration is low (0.5-fold adult concentration) during the first postnatal week and then increases to 3-fold of adult levels between days 14-60. Controlled cortical impact (CCI) at postnatal day (PND) 17, but not PND 7, caused an additional 3-fold increase in COX-2 content and was associated with an increase in the COX-2 product $\mathrm{PGE}_{2}$. Treatment with the COX-2 inhibitor SC58125 in PND17 rats exposed to $\mathrm{CCI}$ attenuated the rise in $\mathrm{PGE}_{2}$ but did not attenuate lesion volume or improve performance in the Morris water maze. (Pediatr Res 62: 271-276, 2007)
\end{abstract}

$\mathrm{C}^{\mathrm{ox}}$ $\mathrm{OX}$ is the rate-limiting enzyme in the production of prostaglandins from arachidonic acid. There are two COX isoforms, COX-1 and COX-2. COX-1 is a constitutive isoform produced in many tissues including stomach, kidneys, blood vessels, and platelets. COX-2 is an inducible isoform found in inflammatory cells and brain; it is the predominant isoform in brain. In brain, COX-2 is induced by synaptic activity and COX-2-generated prostaglandins are important physiologic regulators for a range of activities including local cerebral blood flow and learning. The recent controversy surrounding the association of stroke and COX-2 inhibitors in otherwise healthy patients reinforces the notion that COX-2 has an important role in cerebrovascular homeostasis. On the other hand, overexpression of COX-2 can be harmful. Under pathologic conditions including trauma, ischemia, and chronic neurodegenerative conditions, COX-2 activity is up-regulated, producing reactive oxygen species and toxic prostaglandin

Received July 28, 2006; accepted March 30, 2007.

Correspondence: Robert W. Hickey, M.D., Children's Hospital of Pittsburgh, Division of Pediatric Emergency Medicine, 3705 Fifth Avenue, Pittsburgh, PA 15213; e-mail: robert.hickey@chp.edu

Supported by National Institutes of Health (NIH)/National Institute of Child Health and Human Development grant KO8 HD40848 (RWH); University of Pittsburgh Medical Center, Competitive Medical Research Fund (RWH); NIH/National Institute of Neurological Disorders and Stroke grant RO1 NS42298 (PDA). metabolites that worsen injury [reviewed in (1)]. Accordingly, experimental models of ischemic brain injury show less damage in rodents treated with COX-2 inhibitors and in COX-2 knock out mice.

Both COX-1 (2) and COX-2 are increased after traumatic brain injury (3-5). However, treatment using COX-2 inhibitors in models of traumatic brain injury have led to contradictory results, with some showing a benefit to therapy (6-9) and others showing harm or no benefit $(3,10)$.

COX-2 in the immature brain is developmentally regulated. In the rodent, there is very little COX-2 expression immediately after birth. Expression then increases to achieve peak values during the third and fourth postnatal weeks before decreasing to the intermediate levels contained in adult brain $(11,12)$. A similar pattern of expression is found for nitric oxide synthase (NOS). NOS, like COX, is an important modulator of neuronal function under physiologic conditions and is a paradoxically toxic molecule under pathologic conditions. The expression pattern of COX and NOS, with a peak at approximately $1 \mathrm{mo}$, parallels cortical maturation and synaptogenesis, suggesting that they are potentially important regulators of normal neuronal development. Indeed, abnormal distribution of COX-2 has been described in human children with the neurodegenerative disease Rett syndrome (11). On the other hand, NOS and COX-2 have been shown to act synergistically to worsen ischemic brain injury (13). Thus, under pathologic conditions, the combined peak of $\mathrm{COX}-2$ and NOS expression in maturing brain can create a pathobiologic milieu that may exacerbate brain injury.

The effect of TBI upon COX-2 expression in developing brain and the use of COX-2 inhibition as treatment for TBI in developing animals have not been investigated. In this study, we examine the effects of TBI upon rats before and during peak constitutive COX-2 expression (PND 7 and PND17, respectively). We confirm that COX-2 expression is negligible in the newly born rat and peaks at 3-4 wk. We show that COX-2 is induced by TBI in PND 17 but not PND 7 rats. Finally, we compare prostaglandin production, lesion size, and

Abbreviations: CCI, controlled cortical impact; COX, cyclooxygenase; PND, postnatal day; MWM, Morris water maze; TBI, traumatic brain injury; ROD, relative optic density 
MWM performance in PND17 rats with TBI treated with or without a COX-2 inhibitor.

\section{METHODS}

Chemicals and reagents. The following chemicals and reagents were used: SC58125, COX-2 polyclonal antibody, $\mathrm{PGE}_{2}$ EIA kit (Cayman Chemical, Ann Arbor, MI), COX-2 MAb (Stressgen, Victoria, BC, Canada) and chemiluminescence protein detection kit (Amersham, Arlington Heights, IL).

Animals and treatments. This protocol was approved by the Institutional Animal Care and Use Committee. Brains from naïve Sprague-Dawley (Harlan) rats aged 2, 7, 14, 21, and $30 \mathrm{~d}$ were removed to determine the peak age for constitutive COX-2 protein content using Western blot (see below).

The previously described CCI protocol for adult rats (14) was modified for use in PND 7 (15-20 g) and PND 17 male rats (35-40 g). Using continuous nose cone administration of $2.0 \%$ isoflurane anesthesia in $\mathrm{N}_{2} \mathrm{O} / \mathrm{O}_{2}(2: 1)$, a craniotomy was made in the left parietal bone. CCI was produced using either a 3- or 6-mm diameter impact tip for PND 7 and PND 17 rats, respectively. Impact velocity was $4 \mathrm{~min} / \mathrm{s}$ with a deflection depth of $1.75 \mathrm{~mm}$ or $2.0 \mathrm{~mm}$ for PND 7 and PND 17 rats, respectively. Temperature was maintained at $37-38^{\circ} \mathrm{C}$ (rectal). Following CCI, the wound was closed and anesthesia discontinued. The surgery time for this model is $15-25 \mathrm{~min}$. Rats were recovered in a warmed cubicle for $30 \mathrm{~min}$ and then transferred back to their dams. Rectal temperature was measured at $4,7,24,31$, and $48 \mathrm{~h}$ post injury. The COX-2 inhibitor SC58125 (1-[(4-methylsulfonyl)phenyl]-3-trifluoromethyl-5-[(4-gluroro)phenyl\}pyrazole) was administered to selected rats using an oral dose of $30 \mathrm{mg} / \mathrm{kg}$ at $15 \mathrm{~min}$ and $24 \mathrm{~h}$ after CCI. SC58125 is a selective COX-2 inhibitor that competes with arachidonic acid for the active site of COX-2. It has a half-life of greater than $24 \mathrm{~h}$ with an $\mathrm{IC}_{50}$ for COX-2 that is 100 -fold less than its $\mathrm{IC}_{50}$ for COX-1.

Western blot. Whole brain homogenates were used to determine agerelated differences in constitutive COX-2 expression. Brains were divided into injured and contralateral hemispheres to assess for COX-2 induction. Tissue was lysed in lysis buffer containing SDS, glycerol, $1 \mathrm{M}$ Tris- $\mathrm{HCl}(\mathrm{pH} \mathrm{6.8)} \mathrm{and}$ mini-complete cocktail. Samples (50 $\mu \mathrm{g}$ protein per lane) were separated and blotted onto a polyvinylidene diflouride membrane. Membranes were incubated with monoclonal COX-2 antibody $(1: 1000)$ overnight at $4^{\circ} \mathrm{C}$. Secondary antibody $(1: 1000)$ was incubated for $2 \mathrm{~h}$ at room temperature.

Immunohistochemistry. Brains were removed, placed in formalin at $4^{\circ} \mathrm{C}$ for $48-72 \mathrm{~h}$, and then imbedded in paraffin. Sections $(5 \mu \mathrm{m})$ were cut with a rotary microtome. Immunohistochemistry was performed using the DAB R \& D Systems (Minneapolis, MN) tissue staining kit. The primary antibody COX-2 (polyclonal) diluted 1:50 was incubated overnight at $4^{\circ} \mathrm{C}$. The following day, the secondary antibody, streptavidin reagents, and DAB chromogen were applied as recommended. Slides were counterstained with hematoxylin.

Measurement of prostaglandin E2. Brains were placed in a matrix and a 1-mm cross-section of brain at the midpoint of contusion was removed and immediately placed into liquid nitrogen. Tissue was homogenized in $1 \mathrm{M}$ Tris-HCl ( $\mathrm{pH}$ 6.8)/methanol solution (1:3) on ice. Samples were spun at $14,000 \mathrm{rpm}$ for $30 \mathrm{~min}$ and supernatant was dried via speed vac (Thermoelectron, Marietta, $\mathrm{OH})$. Samples were resuspended in EIA buffer and plated as described by the manufacturer. Plates were incubated overnight at $4^{\circ} \mathrm{C}$. Ellmans reagent from the EIA kit was applied and the plates were read on a plate reader.

Brain weight and lesion size. Brains were weighed wet after removal of olfactory bulbs and cerebellum. Lesion size was determined by measuring hemispheric and lesion areas through the center of the lesion using the MCID Analysis system (GE Healthcare Bio-Sciences Corp., Piscataway, NJ).

Cognitive performance $(\boldsymbol{M W M})$. A MWM task variant was used to compare acquisition rates among groups. The maze consisted of a plastic pool $(180 \mathrm{~cm}$ in diameter) filled with water to a depth of $28 \mathrm{~cm}$, with a clear Plexiglas stand $(10 \mathrm{~cm}$ in diameter and $2 \mathrm{~cm}$ below the water's surface) used as the hidden goal platform. The pool was located in a $2.5 \times 2.5-\mathrm{m}$ room with extra-maze cues that remained constant throughout the experiment. Water maze testing began on postoperative $\mathrm{d} 10$ to allow for recovery from the effects of anesthesia, incisions, and the acute injury. The rats were given four trials per day for five consecutive days. For each daily block of four trials, rats were placed in the pool facing the wall. Trials were initiated from each of four possible start locations in a randomized manner. The location of the goal platform was kept constant. A maximum of $120 \mathrm{~s}$ was allowed for each rat to find the hidden platform. If the rat failed to find the platform within the allotted time, it was placed back on the platform for $30 \mathrm{~s}$ before removal. In between trials, rats were placed in a heated incubator (4-min intertrial interval). As a control for nonspecific deficits such as visual processing and motivation, additional trials were performed with the swim platform visible (2 $\mathrm{cm}$ above the water surface).
Sample sizes and statistical analysis. A total of 128 rats were used. Four rats per age group were used for Western blots of constitutive COX-2 in naïve, developing rats (total 28). Eleven injured and 8 control rats were used for Western blots of induced COX-2. Two to three rats per group were used for immunohistochemistry (total 10). Two to five rats per time point in each group were used for prostaglandin $\mathrm{PGE}_{2}$ assay (total 36). Brain weight, lesion size, and MWM performance were performed on the same cohort of rats using 5-10 per group (total 35). ANOVA was used for between group comparisons with ROD, prostaglandin ELISA, lesion size, and brain weight. Tukey post hoc analysis was used for subgroup comparisons. Repeated measures analysis was used for MWM score, body weight, and temperature.

\section{RESULTS}

COX-2 expression. Western blot analysis of whole brain homogenates from naïve rats shows constitutive COX-2 is increased 2-3-fold in rats from 14 to $30 \mathrm{~d}$ compared with very young (PND 57 ) or mature (PND 90) rats (Fig. 1). Western blot analysis from ipsilateral and contralateral hemispheres at $24 \mathrm{~h}$ in rats injured with CCI show a significant increase in COX-2 in the ipsilateral hemisphere of PND 17 rats $(p=0.03$ post hoc testing with naïve and sham rats pooled as control) but not PND 7 rats (Fig. 2). ROD of injured PND 17 was greater than injured PND $7(p=0.001)$. Figure 3 shows representative COX-2 immunohistochemistry. Although the amount of tissue loss is severe in both PND 7 and PND 17 rats, only the PND 17 rats show increased COX-2 staining compared with naïve rats. Staining is most pronounced in cell bodies of the hippocampus, retrosplenial cortex, perilesional cortex, and lateral cortex.

$\boldsymbol{P} \boldsymbol{G} \boldsymbol{E}_{2}$ content. After determining that PND 17 rats had robust expression of COX-2 that was further increased after $\mathrm{CCI}$, the activity of COX-2 was confirmed by measurement of the COX-2 product Prostaglandin $\mathrm{E}_{2}\left(\mathrm{PGE}_{2}\right)$ in PND 17 rats (Fig. 4). ANOVA revealed significant effects of group ( $p=$ $0.002)$ and sacrifice time $(p=0.021)$. The interaction between group and sacrifice time was not significant. $\mathrm{PGE}_{2}$ was increased approximately 4 -fold at $24 \mathrm{~h}$ in rats with CCI compared with sham-controls. Oral administration of the COX-2 inhibitor SC58125 at $15 \mathrm{~min}$ and $24 \mathrm{~h}$ after CCI attenuated the

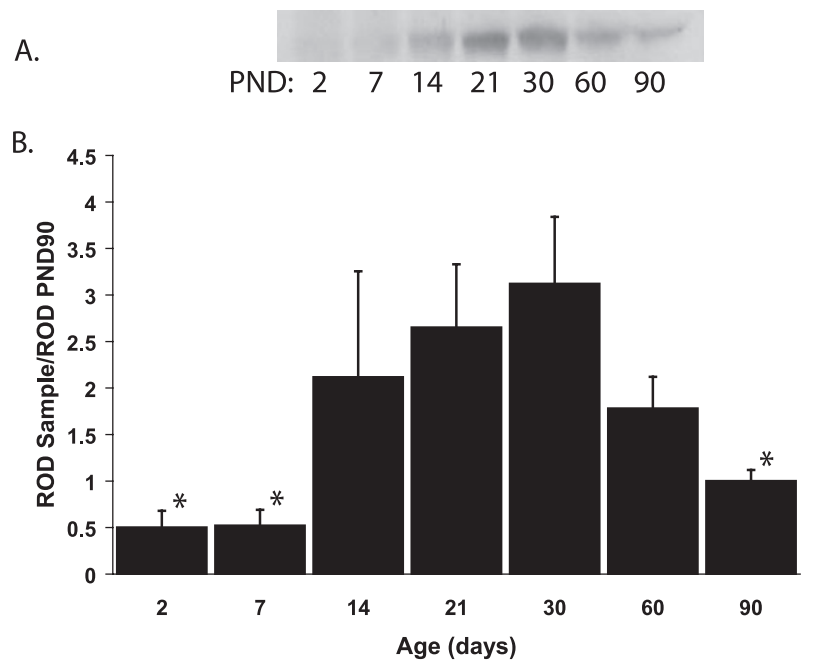

Figure 1. Constitutive COX-2 protein expression. (A) Representative Western blot from whole brain homogenates at different ages in naïve rats. $(B)$ ROD values (mean $\pm 1.0 \mathrm{SE}$ ) from Western blots. Values were standardized to ROD from mature PND 90 within the same gel. ( $n=4$ /group). 
$L \quad R L R L R L R L R L R L R L R L R \quad C t$
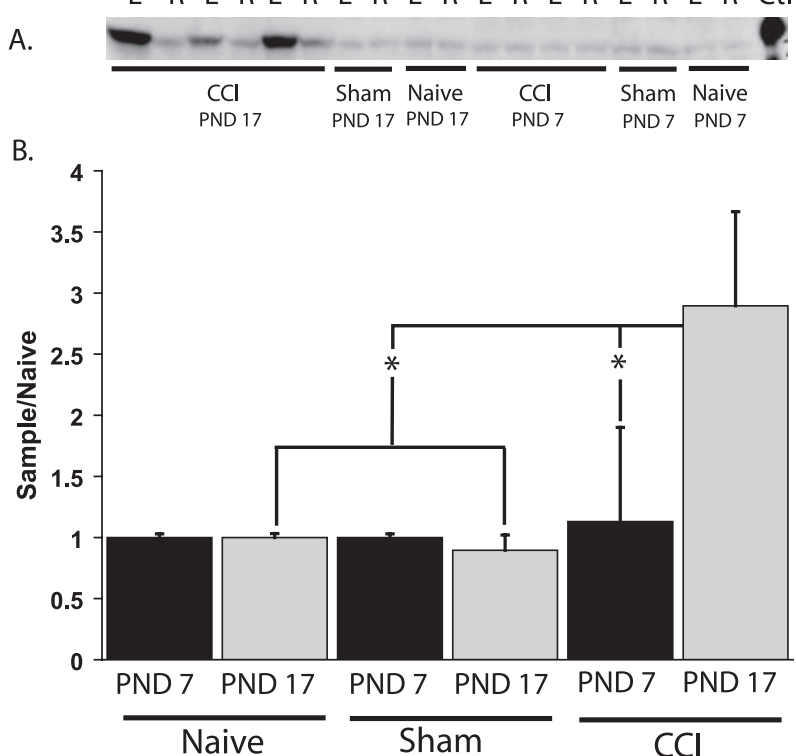

Figure 2. COX-2 protein expression following CCI. (A) Representative Western blot showing increased expression in ipsilateral $(\mathrm{L}=$ left $)$ hemisphere at $24 \mathrm{~h}$ following CCI in PND 17, but not PND 7 rats $(\mathrm{R}=$ right, contralateral sample). Control samples were from sham or naïve rats. $(B)$ ROD values (mean $\pm 1.0 \mathrm{SE}$ ) from ipsilateral hemisphere of injured (CCI) and control PND 7 and PND 17 rats. Values were standardized to ROD from naïve rats of the same age within the same gel (CCI, $n=11$; naïve, $n=4$; sham, $n=4)$ ) * $p<0.05$.

increase $\mathrm{PGE}_{2}$ measured at 24 and $48 \mathrm{~h}$ (statistically significant only at $48 \mathrm{~h} ; p=0.008$ ). Thus, SC58125 was pharmacologically effective within the CNS during the period of maximal COX-2 activity, as measured by $\mathrm{PGE}_{2}$.

Blockade of COX-2 activity. After determining that COX-2 activity could be attenuated with SC58125 treatment following CCI in PND17 rats, the effect of SC58125 treatment upon lesion size and cognitive deficit was assessed. Control groups included naïve rats, sham CCI (anesthetic, craniotomy, no CCI), and CCI + methylcellulose (vehicle for SC58125). Rats

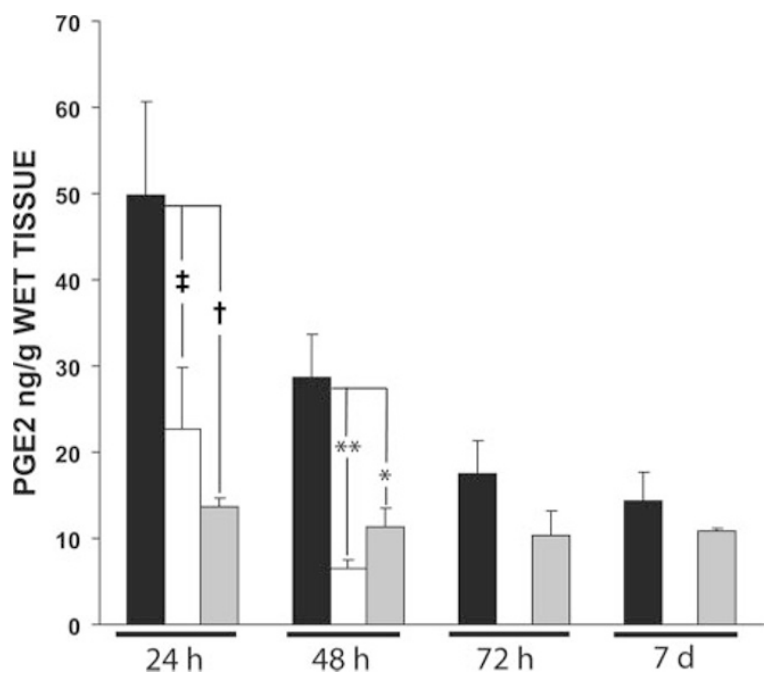

Figure 4. Prostaglandin $\mathrm{E}_{2}$ content. ELISA of $\mathrm{PGE}_{2}$ content (mean \pm 1.0 $\mathrm{SE}$ ) from rats treated with CCI plus vehicle (solid bars, $n=5$ per time point), CCI plus SC58125 (open bars, $n=4$ per time point at 24 and $48 \mathrm{~h}$; not tested at $72 \mathrm{~h}$ or $7 \mathrm{~d}$ ) and sham controls (shaded bars, $n=2$ per time point). Note that $\mathrm{PGE}_{2}$ was not analyzed after $48 \mathrm{~h}$ in SC58125 rats. * $p=0.07$; ** $p=$ $0.01 ; \dagger p=0.12 ; \ddagger p=0.15$.

were recovered for $10 \mathrm{~d}$ before MWM testing. They were killed for measurement of lesion size upon completion of MWM testing at PND 45. Temperature was measured at injury and at $4,7,24$, and $31 \mathrm{~h}$ post injury. No rats developed fever $($ Tmax $=37.8 \mathrm{C})$ and there was no effect of SC58125 upon temperature. Rats were weighed daily; there was no effect of SC58125 upon weight gain. Wet brain weight did not differ between groups at sacrifice (Table 1). Similarly, the size of the lesion was not attenuated by SC58125 treatment (Table 1).

MWM performance. MWM testing during the first $5 \mathrm{~d}$ (with hidden platform) showed that CCI + SC58125 had improved performance compared with CCI rats, but not $\mathrm{CCI}+$ vehicle rats, on $\mathrm{d} 2$ and 3 (ANOVA $p<0.05$ ) (Fig. 5). In any
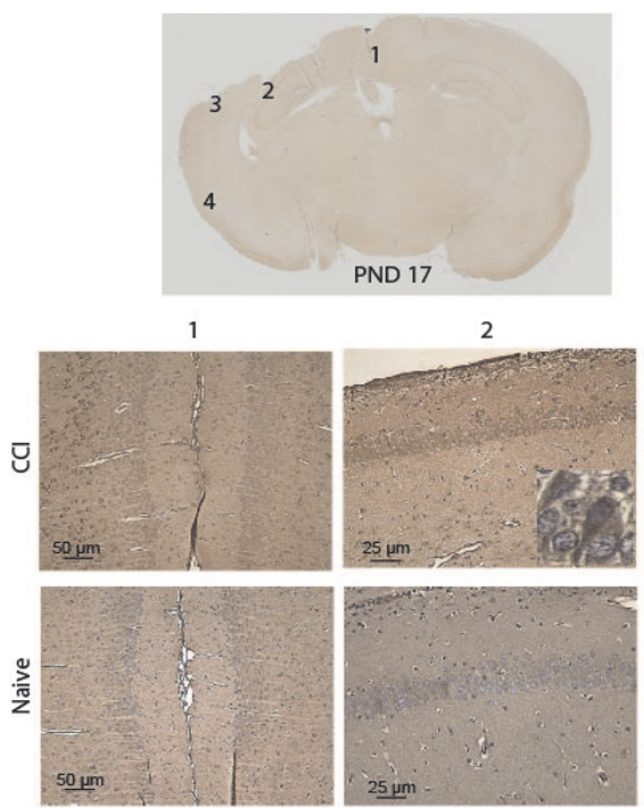

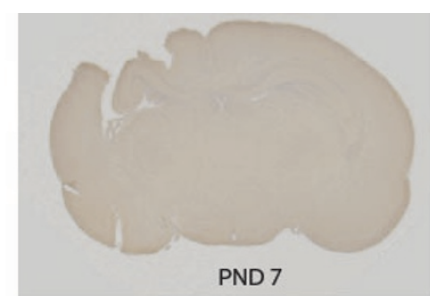

3
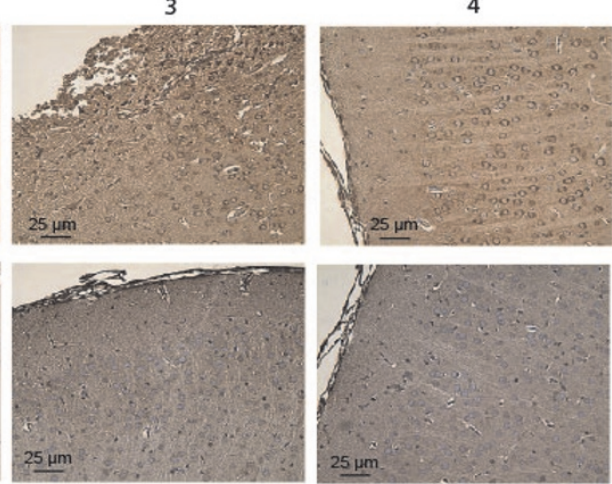

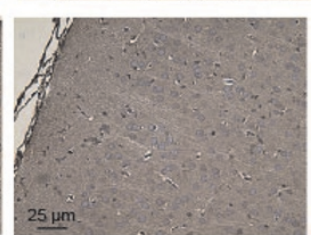

Figure 3. COX-2 immunohistochemistry. (Top) Low-power magnification showing comparable tissue loss at sacrifice in PND 7 and PND 17 rats. (Middle and Bottom) Immunohistochemistry at $24 \mathrm{~h}$ of PND 17 CCI (middle) and PND 17 Naïve (bottom) from brain regions as labeled in the top panel. CCI rats showed increased staining in retrosplenial cortex (region 1, note increased staining in ipsilateral $v s$ contralateral cortex in this image); CA1 hippocampus (region 2, note cellular staining in higher magnification cut out); perilesional cortex (region 3); and lateral cortex (region 4) of PND 17 rats. There was minimal increased staining in PND 7 rats, mostly localized to the perilesional region (not shown). There was no staining in control sections treated with secondary antibody only or with primary antibody quenched with blocking peptide (not shown). 
Table 1. Wet brain weight and lesion size (as measured by the ratio of left to right hemisphere through the impact site) in PND 17 rats at sacrifice (PND 45)

\begin{tabular}{|c|c|c|c|c|c|}
\hline & $\begin{array}{c}\text { Naïve }+ \text { SC58125 } \\
(n=5)\end{array}$ & $\begin{array}{c}\text { Sham }+ \text { SC58125 } \\
(n=5)\end{array}$ & $\begin{array}{c}\text { CCI } \\
(n=5)\end{array}$ & $\begin{array}{c}\text { CCI }+ \text { vehicle } \\
(n=5)\end{array}$ & $\begin{array}{c}\mathrm{CCI}+\mathrm{SC} 58125 \\
(n=10)\end{array}$ \\
\hline Brain weight $(\mathrm{g})$ & $1.59(0.07)$ & $1.64(0.07)$ & $1.56(0.02)$ & $1.53(0.07)$ & $1.59(0.07)$ \\
\hline Area left/right hemisphere & $1.0(0.01)$ & $1.0(0.01)$ & $0.73(0.03)^{*}$ & $0.83(0.04)^{*}$ & $0.80(0.02)^{*}$ \\
\hline
\end{tabular}

Data are given as mean $(\mathrm{SEM}) . * p<0.05$ compared with naïve and sham rats.

case, this difference was not sustained on $\mathrm{d} 4$ and 5 of testing. MWM performance on $\mathrm{d} 7$ and 8 of testing (with the platform visible) showed equally improved times in all groups suggesting that visual acuity and motor function was not impaired.

\section{DISCUSSION}

This study confirms that constitutive expression of COX-2 is developmentally regulated (with peak expression occurring at the same time as peak synaptogenesis in the rat) and is the first to show that COX-2 induction in response to brain injury is also developmentally regulated. We found an increase in COX-2 expression in PND 17, but not PND 7 rats, following CCI. A similar result has been reported by Tocco et al. (12) using a model of kainate-induced seizures; kainate induced intense seizures in PND 7, 14, 21, 28 and 60 rats but COX-2 mRNA was increased only in rats aged $>$ PND 7 . Both studies highlight the importance of examining mechanisms of injury and testing neuroprotective strategies across a spectrum of ages.

Developmental susceptibility to injury is likely related to the physiology of brain maturation including proliferation, migration, differentiation, synaptogenesis, myelination, and apoptosis. With respect to these events, the PND 7 rat has a relative developmental age of a newly born full-term human and the PND 17 rat has a relative developmental age of a toddler (PND 35-45 is adolescence). At the cellular level, pathways involved in programmed cell death, including caspase activation and classic apoptotic morphology, are relatively more active and more easily induced in very young (PND $\leq 7$ rats) compared with older maturing or adult rats.

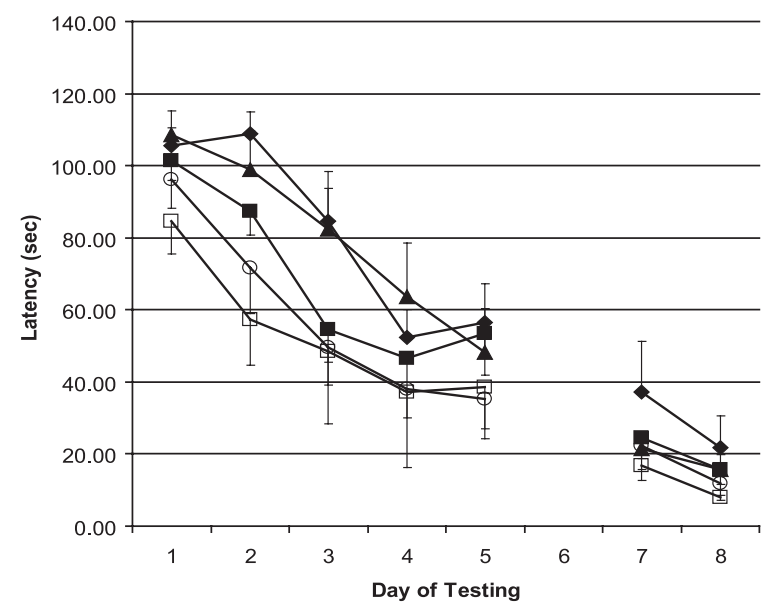

Figure 5. MWM performance. Data are shown for mean and SEM for each day of testing. The platform was hidden during trials on $\mathrm{d} 1-5$ and was visible on d 7-8. $\bullet$, CCI; $\boldsymbol{\square}$, CCI + SC58125; $\mathbf{\Delta}$, CCI + Vehicle; $\square$, Naive + SC58125; O, Sham + SC58125.
Maturation of excitatory neurotransmitter receptors (function, density, and distribution) peaks slightly later (PND 14-21) and is associated with increased susceptibility to hypoxicischemic injury $(15,16)$. The maturation of excitatory neurotransmitter receptors coincides with a peak in constitutive expression of both COX-2 $(11,12)$ and NOS. COX and NOS have been shown to act synergistically to contribute to brain injury after cerebral ischemia (13). NOS has previously been reported to increase following TBI in immature rats (17). This is the first report of COX-2 induction following TBI in an immature animal.

There are several potential mechanisms whereby COX-2 might exacerbate neuronal injury. COX-2 converts arachidonic acid to $\mathrm{PGH}_{2}$ via a cyclooxygenase followed by a peroxidase conversion that can generate free radicals (18) and exacerbate reperfusion injury (13). $\mathrm{PGH}_{2}$ is then metabolized by specific prostaglandin synthases into the first-order prostaglandins $\mathrm{PGA}_{2}, \mathrm{PGD}_{2}, \mathrm{PGE}_{2}, \mathrm{PGI}_{2}$, and thromboxane. The role of specific prostaglandins in brain injury is complicated by the diversity and distribution of prostaglandin receptors. Accordingly, some studies show prostaglandin-specific neurotoxic effects (19-21) whereas others show neuroprotective effects (22-24). This is further complicated by changes in the distribution of specific prostaglandin synthases during brain maturation (25). Recently, a series of nonenzymatic metabolites of prostaglandins (cyclopentenone prostaglandins) have been shown to trigger neuronal apoptosis $(26,27)$ and perturb ubiquitin-dependent protein processing (28). Cyclopentenone derivatives of $\mathrm{PGD}_{2}$ have been reported following TBI (4). Thus, there are several potential mechanisms of COX-2 toxicity that could be relevant to TBI.

Although we found a robust increase in COX-2 following CCI in PND17 rats and there are biologically plausible reasons, as well as some experimental data, invoking a detrimental role for COX-2 in TBI, we found no protection using the COX-2 inhibitor SC58125. Our results are consistent with those reported by Dash et al. (3) and Koyfman et al. (10) but are in opposition to those reported by Cernak et al. (7), Gopez et al. (9), and Moochhala et al. (8). These disparate results might be caused by differences in models (CCI, fluidpercussion, and weight drop), treatment protocols (pre- or postinjury initiation of drugs, duration of therapy), specificity of drugs (e.g., Moochhala administered a combined NOS and COX-2 inhibitor), and outcome measures (duration of follow up, histology techniques, motor or cognitive functional tests). Table 2 compares these variables across studies: no clear pattern emerges that would explain the difference between the "positive" and "negative" studies. 
Table 2. Comparison of studies examining treatment of TBI with COX-2 inhibition

\begin{tabular}{|c|c|c|c|c|}
\hline $\begin{array}{l}\text { Study } \\
\text { (ref no.) }\end{array}$ & Animal & Injury & Drug protocol & Outcome and effect \\
\hline 6 & $\begin{array}{l}\text { Sprague-Dawley } \\
\text { adult males }\end{array}$ & Diffuse TBI & $\begin{array}{l}\text { Nimesulide IP } 30 \text { min post-injury and } \\
\text { daily } \times 10 \mathrm{~d}\end{array}$ & Cognitive (Barnes maze): $\uparrow$ Motor (rotarod): $\leftrightarrow$ \\
\hline 8 & $\begin{array}{l}\text { Sprague-Dawley } \\
\text { adult males }\end{array}$ & $\begin{array}{l}\text { Fluid-percussion (rapid } \\
\text { epidural saline injection) }\end{array}$ & $\begin{array}{l}\text { Mercaptoethylguanidine }(\mathrm{COX}-2 \text { and } \\
\text { iNOS inhibitor and peroxynitrite } \\
\text { scavenger) IP } 1 \mathrm{~h} \text { post-injury }\end{array}$ & $\begin{array}{l}\text { Motor (rotarod): } \uparrow \text { Neuroscore: } \uparrow \text { Motor (grip } \\
\text { strength): } \uparrow \text { Histology (NISSL stain): } \\
\uparrow \text { Cerebral perfusion: } \uparrow\end{array}$ \\
\hline 3 & $\begin{array}{l}\text { Long-Evans adult } \\
\text { males }\end{array}$ & Lateral cortical impact & $\begin{array}{l}\text { Celecoxib PO } 15 \text { min pre-injury and } \\
\text { bid } \times 5 \mathrm{~d}\end{array}$ & $\begin{array}{l}\text { Cognitive (MWMze and conditioned emotional } \\
\text { response): } \leftrightarrow \text { Neuroscore: } \leftrightarrow \text { Motor (beam } \\
\text { balance, flexion reflex, paw placement): } \downarrow\end{array}$ \\
\hline
\end{tabular}

$\uparrow=$ benefit, $\downarrow=$ harm, $\leftrightarrow=$ no effect.

A strength of this study is that we used a clinically relevant postinjury treatment design and confirmed that the inhibitor had a pharmacologic effect in brain. We used $\mathrm{PGE}_{2}$ content as a surrogate marker for COX-2 activity. We chose to measure $\mathrm{PGE}_{2}$ based upon our previous success with the $\mathrm{PGE}_{2}$ ELISA in a variety of experimental paradigms. $\mathrm{PGD}_{2}$ and $\mathrm{PGE}_{2}$ are the predominant prostaglandins in brain. We assume, but did not confirm, that changes in $\mathrm{PGE}_{2}$ reflect changes in other members of the prostaglandin family. We chose a short duration of therapy $(24 \mathrm{~h})$ and confirmed that $\mathrm{PGE}_{2}$ did not "rebound" at $48 \mathrm{~h}$ ( $24 \mathrm{~h}$ post dosing). The duration of COX-2 inhibition is an important consideration because there is data in rat models of inflammation showing that the prostaglandin response evolves over time from an early pro-inflammatory to a delayed anti-inflammatory (reparatory) phase and thus a short duration of therapy targets the early pro-inflammatory response. Whether or not a similar change from prostaglandinmediated inflammation to repair occurs following TBI has not been studied. We looked at $\mathrm{PGE}_{2}$ content at $72 \mathrm{~h}$ and $7 \mathrm{~d}$ following CCI to determine the duration of trauma-mediated COX-2 activity. We found that $\mathrm{PGE}_{2}$ was slightly elevated at both time points but the difference was not statistically different. This is consistent with the early $(24 \mathrm{~h})$ peak of $\mathrm{PGE}_{2}$ reported in other models of TBI $(8,9)$. However, the prolonged increase in COX-2 mRNA ( 3 d) and COX-2 immunohistochemistry ( $7 \mathrm{~d}$ ) reported by others (5) suggests that COX-2 activity remains elevated, but below the threshold of our $\mathrm{PGE}_{2}$ ELISA. Thus, COX-2 may continue to have effects at the cellular level beyond the duration of inhibition provided by the $24 \mathrm{~h}$ dose of NS58125.

Unlike Gopez et al. (9), but like Dash and co-workers (3), we found no difference in MWM performance in treated rats. This may be due to differences in the scoring system used to assess maze performance. Alternatively, COX-2 might be particularly important during synaptogenesis in the rat and inhibition in the PND17 rat might interfere with development of normal connectivity. Along these lines, Kaufmann et al. (11) describe COX-2 immunoreactivity expression in a developmental pattern that parallels the crucial period for somatosensory and visual cortex development in the rat. They also demonstrate that COX-2 immunoreactivity is altered in patients with Rhett syndrome, a syndrome characterized by mental retardation, neurodegeneration, and altered dendritic maturation. Also, Teather et al. (29) describe impaired memory consolidation in rats treated with COX-2 inhibition during MWM training. Finally, COX-2 over expressing transgenic mice develop an age-dependent deficit in spatial memory (30). Thus, chronic COX-2 inhibition or acute COX-2 inhibition immediately before a memory task might impair performance. It is less likely that a short course of COX-2 inhibition administered many days before testing, as occurred in this protocol, would have similar effects. On the other hand, there is evidence that even very brief inhibition of excitatory neurotransmitters in immature rats (e.g. a single dose of the NMDA inhibitor MK801 in PND 7 rats) can trigger diffuse apoptosis. Additional studies are needed to determine whether maturing PND17 rats are especially susceptible to memory impairment caused by COX-2 inhibition.

One limitation of this study is that we did not measure products of arachidonic acid that are unrelated to COX-2 activity. Specifically, COX-2 inhibition may shunt arachidonic acid metabolism into the lipoxygenase pathway that generates HETES and leukotrienes. Some of these lipoxygenase derivatives are neurotoxic while others are protective. A study by Gopez et al. (9) found that DFU treatment attenuated the increase in HETE while amplifying the production of EET following TBI (the authors speculate that this profile inhibits inflammation).

Another limitation of this study is that the tip diameter and depth of impact in the CCI device was greater for the PND 17 rats compared with the PND 7 rats. It was necessary to use a relatively larger force in the PND 17 rats to cause a similar amount of tissue loss. Tissue loss was substantial in both PND 7 and PND 17 rats (Fig. 3). Because both paradigms resulted in similar tissue injury, the absence of COX-2 induction in the PND 7 rat was unlikely to be due to the differences in applied forces. Also, we did not see a difference in lesion size or brain weight in PND 17 rats treated with SC58125. It is possible that a more sensitive and robust assay of lesion size (stereometric volume measurement) might detect a difference, but, to date, no studies of COX-2 inhibition following TBI have reported a tissue sparing effect.

In summary, this is the first study to report that COX-2 induction following TBI is developmentally regulated. We 
were unable to show a neuroprotective effect of COX-2 inhibition. There are several potential explanations for the lack of neuroprotection in this study. Specifically, the lesion might be too severe to be manipulable, the inhibition protocol might be insufficiently protective, the chosen outcomes might be insensitive to small beneficial effects, or the developmental requirement of COX-2 for normal development might outweigh the pathologic contribution.

\section{REFERENCES}

1. Graham SH, Hickey RW 2003 Cyclooxygenases in central nervous system diseases: a special role for cyclooxygenase 2 in neuronal cell death. Arch Neurol 60:628-630

2. Schwab JM, Seid K, Schluesener HJ 2001 Traumatic brain injury induces prolonged accumulation of cyclooxygenase- 1 expressing microglia/brain macrophages in rats. J Neurotrauma 18:881-890

3. Dash PK, Mach SA, Moore AN 2000 Regional expression and role of cyclooxygenase-2 following experimental traumatic brain injury. J Neurotrauma 17:69-81

4. Kunz T, Marklund N, Hillered L, Oliw EH 2002 Cyclooxygenase-2, prostaglandin synthases, and prostaglandin $\mathrm{H} 2$ metabolism in traumatic brain injury in the rat. J Neurotrauma 19:1051-1064

5. Strauss KI, Barbe MF, Marshall RM, Raghupathi R, Mehta S, Narayan RK 2000 Prolonged cyclooxygenase- 2 induction in neurons and glia following traumatic brain injury in the rat. J Neurotrauma 17:695-711

6. Cernak I, O'Connor C, Vink R 2002 Inhibition of cyclooxygenase 2 by nimesulide improves cognitive outcome more than motor outcome following diffuse traumatic brain injury in rats. Exp Brain Res 147:193-199

7. Cernak I, O'Connor C, Vink R 2001 Activation of cyclo-oxygenase-2 contributes to motor and cognitive dysfunction following diffuse traumatic brain injury in rats. Clin Exp Pharmacol Physiol 28:922-925

8. Moochhala SM, Lu J, Xing MC, Anuar F, Ng KC, Yang KL, Whiteman M, Atan S 2005 Mercaptoethylguanidine inhibition of inducible nitric oxide synthase and cyclooxygenase- 2 expressions induced in rats after fluid-percussion brain injury. J Trauma 59:450-457

9. Gopez JJ, Yue H, Vasudevan R, Malik AS, Fogelsanger LN, Lewis S, Panikashvili D, Shohami E, Jansen SA, Narayan RK, Strauss KI 2005 Cyclooxygenase-2-specific inhibitor improves functional outcomes, provides neuroprotection, and reduces inflammation in a rat model of traumatic brain injury. Neurosurgery 56:590-604

10. Koyfman L, Kaplanski J, Artru AA, Talmor D, Rubin M, Shapira Y 2000 Inhibition of cyclooxygenase 2 by nimesulide decreases prostaglandin E2 formation but does not alter brain edema or clinical recovery after closed head injury in rats. J Neurosurg Anesthesiol 12:44-50

11. Kaufmann WE, Worley PF, Taylor CV, Bremer M, Isakson PC 1997 Cyclooxygenase-2 expression during rat neocortical development and in Rett syndrome. Brain Dev 19:25-34

12. Tocco G, Freire-Moar J, Schreiber SS, Sakhi SH, Aisen PS, Pasinetti GM 1997 Maturational regulation and regional induction of cyclooxygenase-2 in rat brain: implications for Alzheimer's disease. Exp Neurol 144:339-349

13. Nogawa S, Forster C, Zhang F, Nagayama M, Ross ME, Iadecola C 1998 Interaction between inducible nitric oxide synthase and cyclooxygenase- 2 after cerebral ischemia. Proc Natl Acad Sci USA 95:10966-10971
14. Dixon CE, Clifton GL, Lighthall JW, Yaghmai AA, Hayes RL 1991 A controlled cortical impact model of traumatic brain injury in the rat. J Neurosci Methods 39:253-262

15. McDonald JW, Johnston MV 1990 Physiological and pathophysiological roles of excitatory amino acids during central nervous system development. Brain Res Brain Res Rev 15:41-70

16. Towfighi J, Mauger D, Vannucci RC, Vannucci SJ 1997 Influence of age on the cerebral lesions in an immature rat model of cerebral hypoxia-ischemia: a light microscopic study. Brain Res Dev Brain Res 100:149-160

17. Clark RS, Kochanek PM, Schwarz MA, Schiding JK, Turner DS, Chen M, Carlos TM, Watkins SC 1996 Inducible nitric oxide synthase expression in cerebrovascular smooth muscle and neutrophils after traumatic brain injury in immature rats. Pediatr Res 39:784-790

18. Jiang J, Borisenko GG, Osipov A, Martin I, Chen R, Shvedova AA, Sorokin A, Tyurina YY, Potapovich A, Tyurin VA, Graham SH, Kagan VE 2004 Arachidonic acid-induced carbon-centered radicals and phospholipid peroxidation in cyclooxygenase-2-transfected PC12 cells. J Neurochem 90:1036-1049

19. Bezzi P, Carmignoto G, Pasti L, Vesce S, Rossi D, Rizzini BL, Pozzan T, Volterra A 1998 Prostaglandins stimulate calcium-dependent glutamate release in astrocytes. Nature 391:281-285

20. Manabe Y, Anrather J, Kawano T, Niwa K, Zhou P, Ross ME, Iadecola C 2004 Prostanoids, not reactive oxygen species, mediate COX-2-dependent neurotoxicity. Ann Neurol 55:668-675

21. Kawano T, Anrather J, Zhou P, Park L, Wang G, Frys KA, Kunz A, Cho S, Orio M, Iadecola C 2006 Prostaglandin E(2) EP1 receptors: downstream effectors of COX-2 neurotoxicity. Nat Med 12:225-229

22. Kim EJ, Kwon KJ, Park JY, Lee SH, Moon CH, Baik EJ 2002 Neuroprotective effects of prostaglandin E2 or cAMP against microglial and neuronal free radical mediated toxicity associated with inflammation. J Neurosci Res 70:97-107

23. Takamatsu H, Tsukada H, Watanabe Y, Cui Y, Kataoka Y, Hosoya T, Suzuki M, Watanabe Y 2002 Specific ligand for a central type prostacyclin receptor attenuates neuronal damage in a rat model of focal cerebral ischemia. Brain Res 925:176-182

24. McCullough L, Wu L, Haughey N, Liang X, Hand T, Wang Q, Breyer RM, Andreasson K 2004 Neuroprotective function of the PGE2 EP2 receptor in cerebral ischemia. J Neurosci 24:257-268

25. Ueno R, Osama H, Urade Y, Hayaishi O 1985 Changes of enzymes involved in prostaglandin metabolism and prostaglandin binding proteins in rat brain during development and aging. J Neurochem 45:483-489

26. Kondo M, Shibata T, Kumagai T, Osawa T, Shibata N, Kobayashi M, Sasaki S, Iwata M, Noguchi N, Uchida K 2002 15-Deoxy-Delta(12,14)-prostaglandin J(2): the endogenous electrophile that induces neuronal apoptosis. Proc Natl Acad Sci U S A 99:7367-7372

27. Yagami T, Ueda K, Asakura K, Hayasaki-Kajiwara Y, Nakazato H, Sakaeda T, Hata S, Kuroda T, Takasu N, Hori Y 2002 Group IB secretory phospholipase A2 induces neuronal cell death via apoptosis. J Neurochem 81:449-461

28. Li Z, Melandri F, Berdo I, Jansen M, Hunter L, Wright S, Valbrun D, FigueiredoPereira ME 2004 Delta12-Prostaglandin J2 inhibits the ubiquitin hydrolase UCH-L1 and elicits ubiquitin-protein aggregation without proteasome inhibition. Biochem Biophys Res Commun 319:1171-1180

29. Teather LA, Packard MG, Bazan NG 2002 Post-training cyclooxygenase-2 (COX-2) inhibition impairs memory consolidation. Learn Mem 9:41-47

30. Andreasson KI, Savonenko A, Vidensky S, Goellner JJ, Zhang Y, Shaffer A, Kaufmann WE, Worley PF, Isakson P, Markowska AL 2001 Age-dependent cognitive deficits and neuronal apoptosis in cyclooxygenase-2 transgenic mice. J Neurosci 21:8198-8209 